\title{
AN EXTENSION OF A THEOREM OF BESSAGA
}

\author{
CHARLES A. RILEY ${ }^{1}$
}

ABSTRACT. In [1], C. Bessaga has shown that if $X$ is a linear topological space admitting a weak incomplete norm $w$ and $A \subset X$ is closed in the $w$-completion of $X$, then $A$ is negligible in $X$. The present paper establishes this result in a space admitting a weak incomplete linear metric.

In his proof of the theorem mentioned above, Bessaga translates $A$ to $A+e$, where $e$ is in the $w$-completion of $X$, but outside $X$. We accomplish the same motion in the proof below. Unfortunately the elegance of Bessaga's proof is lost, since the broken line he proposes cannot be constructed in all spaces. A paranorm $w$ in a linear space is a function to the nonnegative reals satisfying:

(1) $w(0)=0$

(2) $w(-x)=w(x)$,

(3) $w(x+y) \leq w(x)+w(y)$,

(4) if $t_{n} \rightarrow t, w\left(x_{n}-x\right) \rightarrow 0$, then $w\left(t_{n} x_{n}-t x\right) \rightarrow 0$.

Every metrizable linear topology is given by a paranorm which is total $(w(x)=0$ implies $x=0)$ and strictly increasing $(0<t<1, x \neq 0$ implies $w(t x)<w(x))$. If $w$ is such a paranorm and $U=\left\{x \mid w\left(x-x_{0}\right)<\epsilon\right\}$, then $U$ is shrinkable at $x_{0}$ in the sense of Ives [2] and Klee [4]. That is, $t \in[0,1)$, $x \in \bar{U}$ implies $x_{0}+t\left(x-x_{0}\right) \in U$.

We state the main result of the paper.

(1) Theorem. Suppose $X$ is a linear topological space and $A \subset X$. If $X$ admits a weak, incomplete, total, strictly increasing paranorm $w$ such that $A$ is closed in the completion of $w$, then $A$ is negligible.

The theorem applies to the familiar spaces of functions on $[0,1]$ (including $\left.L^{p}, 0<p<1\right)$, since these, except for the space of all measurable functions, admit the weak, incomplete paranorm $\int[0,1]|f| /(1+|f|)$. A similar ap-

Received by the editors July 24, 1973 and, in revised form, January 24, 1974.

AMS (MOS) subject classifications (1970). Primary 57A20; Secondary 46A15.

Key words and phrases. Negligibility, shrinkable set, paranorm.

${ }^{1}$ This paper was originally intended as a proof of an isotopy theorem stated in [5]. The author is grateful to the referee for suggesting that the theorem should be true for the larger class of sets considered here. 
plication can be made to sequence spaces, using the paranorm $w(x)=$ $\sum\left|x_{n}\right| / 2^{n}\left(1+\left|x_{n}\right|\right)$.

While we cannot use the distance from $A$ to construct a homeomorphism on the whole space (as Bessaga does), we can, using distance, define homeomorphisms on certain closed subspaces and extend linearly. If $P$ is a closed hyperplane in a normed space and $x \notin P$, then the translates $P_{n}=P+n x \mathrm{di}$ vide the space, and projection in direction $x$ into $P_{n}$ is continuous. A similar division may be effected in a general linear topological space by substituting for $P$ the boundary of a cone over a shrinkable set.

(2) Theorem. Suppose $U$ is an open subset of a linear topological space $Y$ and that $U$ is shrinkable at $x_{0} \neq 0$. Suppose also $0 \notin \bar{U}$ and $C=$ $\{\lambda x \mid x \in U, \lambda>0\}$. Then $C$ is open, and if $y \in \bar{C}, \mu>0$, then $y+\mu x_{0} \in C$. Each line $\left\{z+\lambda x_{0} \mid \lambda \in(-\infty, \infty)\right\}$ intersects $\mathrm{Bd} C$ exactly once.

Proof. It is clear that $C$ is open. For each $c \in C$, choose $\lambda(c)>0$, $u(c) \in U$ so that $c=\lambda(c) u(c)$. Suppose $\lambda$ is unbounded on each neighborhood of $y$. For each neighborhood $W$ of $y$ and each positive intege: $n$, choose $c_{w, n}$ such that $c_{w, n} \in W \cap C$ and $\lambda\left(c_{w, n}\right)>n . u\left(c_{w, n}\right)=\left(1 / \lambda\left(c_{w, n}\right)\right) c_{w, n} \rightarrow 0$, since $c_{w, n} \rightarrow y$. Thus $0 \in \bar{U}$, a contradiction. Now let $W$ be a neighborhood of $y$ on which $\lambda$ is bounded, and suppose $y_{\delta} \rightarrow y$ with $y_{\delta} \in W \cap C$. If $\lambda\left(y_{\delta}\right)$ $\rightarrow 0$, take $\tau$ such that $0<\tau<\mu$. Since $\left(1 / \lambda\left(y_{\delta}\right)\right) y_{\delta} \in U$,

$$
\lambda\left(y_{\delta}\right) \tau^{-1}\left(\left(1 / \lambda\left(y_{\delta}\right)\right) y_{\delta}-x_{0}\right) \in U-x_{0} \text { for } \delta>\delta_{0},
$$

since $U$ is shrinkable at $x_{0}$. Thus $\tau^{-1} y \in \bar{U}-x_{0}$ so that $\mu^{-1} y=(\tau / \mu) \tau^{-1} y \in$ $U-x_{0}$ and $y+\mu x_{0} \in \mu U \subset C$. Otherwise $\lambda\left(y_{\delta}\right)$ has a convergent subnet $\lambda\left(y_{\delta(\alpha)}\right) \rightarrow \lambda_{0}>0$.

$$
u\left(y_{\delta(\alpha)}\right)=\left(1 / \lambda\left(y_{\delta(\alpha)}\right)\right)_{\delta_{(\alpha)}} \rightarrow \lambda_{0}^{-1} y \in \bar{U}
$$

Thus

$$
\left(\lambda_{0} /\left(\lambda_{0}+\mu\right)\right)\left(\lambda_{0}^{-1} y-x_{0}\right) \in U-x_{0},
$$

from which it follows that $y+\mu x_{0} \in\left(\lambda_{0}+\mu\right) U \subset C$. It remains to show that a line $\left\{z+\lambda x_{0}\right\}$ meets $\mathrm{Bd} C$ exactly once. It is now sufficient to show that the line meets both $C$ and the complement of $C$. If for each $n, z+n x_{0} \notin C$, then $x_{0}+n^{-1} z=n^{-1}\left(z+n x_{0}\right) \notin C$ and $x_{0} \notin C$, a contradiction. If for every positive integer $n, z-n x_{0} \in C$, then $n^{-1} z-x_{0} \in C$, so that $-x_{0} \in \bar{C}$. By the first part of the theorem this implies $0 \in C$. 
(3) Theorem. If $C$ is as in (2) and $q(y)=\left\{y+\lambda x_{0} \mid \lambda \in(-\infty, \infty)\right\} \cap \mathrm{Bd} C$ for each $y \in Y$, then $q$ is continuous.

Proof. For $x \in Y$, let $\lambda(x)$ be such that $q(x)=x+\lambda(x) x_{0}$. Suppose that on each neighborhood of $y, \lambda$ is not bounded above. For each neighborhood $W$ of $y$ and each positive integer $n$, choose $y_{w, n} \in W$ such that $\lambda\left(y_{w, n}\right)>n$. Then $y_{w, n}+n x_{0} \notin C$, so that $n^{-1} y_{w, n}+x_{0} \notin C$. This implies $x_{0} \notin C$, a contradiction. We can similarly show $\lambda$ is bounded below on some neighborhood of $y$. Let $W$ be a neighborhood on which $\lambda$ is bounded and $y_{\delta} \rightarrow y$ with $y_{\delta} \in W$. Now suppose $\lambda\left(y_{\delta(a)}\right)$ is a subnet of $\lambda\left(y_{\delta}\right)$ converging to $\lambda_{0}$. Then $q\left(y_{\delta(\alpha)}\right) \rightarrow y+\lambda_{0} x_{0}$. Since $\mathrm{Bd} C$ is closed, $y+\lambda_{0} x_{0} \in \mathrm{Bd} C$. Since $y+$ $\lambda(y) x_{0}$ is also in $\mathrm{Bd} C, \lambda_{0}=\lambda(y)$. That is, every convergent subnet of $\left\{\lambda\left(y_{\delta}\right)\right\}$ converges to $\lambda(y)$. Thus $\lambda\left(y_{\delta}\right) \rightarrow \lambda(y)$, and $q\left(y_{\delta}\right) \rightarrow q(y)$.

(4) Lemma. Suppose $X$ is a Hausdorff linear topological space and $Y a$ closed subset of $X$. If $h: Y \rightarrow X$ is continuous and $1-1$, and there is a compact set $K \subset X$ such that $h(y)-y \in K$ for each $y \in Y$, then $h$ is a topological embedding. If $K$ is also a linear set and $Y=X$, then $h$ is a homeomorphism onto $X$.

Proof. The continuity of $h^{-1}$ needs to be verified. For $x \in h(Y), x=$ $h(y)=y+k$ with $k \in K$, so that $y=h^{-1}(x)=x-k$. Suppose $x_{\delta}, x \in h(Y)$ and $x_{\delta} \rightarrow x \cdot h^{-1}\left(x_{\delta}\right)=x_{\delta}-k_{\delta}$ with $k_{\delta} \in K$. If $k_{\delta(\alpha)} \rightarrow k$ is a convergent subnet, then $h^{-1}\left(x_{\delta(a)}\right)=x_{\delta(a)}-k_{\delta(a)} \rightarrow x-k \in Y$. Thus $x_{\delta(a)}=b h^{-1}\left(x_{\delta(a)}\right) \rightarrow$ $h(x-k)$, so that $x=h(x-k)$ and $h^{-1}(x)=x-k$. This shows every convergent subnet of $\left\{k_{\delta}\right\}$ converges to $k$, and so $h^{-1}\left(x_{\delta}\right)=x_{\delta}-k_{\delta} \rightarrow x-k=$ $h^{-1}(x)$. If $Y=X$, and $K$ is linear, then the intermediate value theorem applies to each line in direction $K$ to show $h$ is onto $X$.

We proceed to the proof of (1). Let $Y$ be the $w$-completion of $X$ and $e \in Y \backslash X$. It is not hard to find a sequence $\left\{z_{n}\right\} \subset X$ such that $z_{n} \rightarrow e, z_{1}$ $=0$ and $w\left(z_{n+1}-z_{n}\right)<w\left(z_{n}-z_{n-1}\right) / 10$ for each $n \geq 2$. Let $x_{n}=z_{n+1}-z_{n}$. We will define a sequence of homeomorphisms whose composition will be the desired mapping. The first will be in direction $x_{1}$ and move points $y$ in a neighborhood of $A$ to $y+x_{1}$. Points far from $A$ will not be moved. We first define the homeomorphism on translates of the boundary of a cone and then fill in linearly. There exists $\lambda$ such that $w\left(x_{1}\right)<w\left(\lambda x_{1}\right)<2 w\left(x_{1}\right)$. With $C$ as the cone of (2) over $\left\{x \in Y \mid w\left(x-x_{1}\right)<w\left(x_{1}\right) / 2\right\}$, let

$$
P_{n}=(\mathrm{Bd} C)+n \lambda x_{1} \text { and } D=\bigcup\left\{P_{n} \mid n \text { an integer }\right\} \text {. }
$$


Let

$$
w_{1}(y)=\min \left\{w(y), w\left(x_{1}\right)\right\} \quad \text { and } \quad A_{1}=\left\{y \in Y \mid d(y, A) \leq 5 w\left(x_{1}\right)\right\} .
$$

For $y \in D$, we take

$$
h_{1}(y)=y+\left(w\left(x_{1}\right)-d_{1}\left(y, A_{1}\right)\right)\left(1 / w\left(x_{1}\right)\right) x_{1} .
$$

Here $d_{1}\left(y, A_{1}\right)=\inf \left\{w_{1}(y-z) \mid z \in A_{1}\right\}_{0} h_{1}$ is extended by requiring that it map $\left[y, y+\lambda x_{1}\right]$ linearly onto $\left[h_{1}(y), h_{1}\left(y+\lambda x_{1}\right)\right]$ for each $y \in D$. Using (2), it is easy to see that $h_{1}$ is thereby defined on all of $Y$. The mapping $h_{1} \mid D$ is clearly 1-1 and continuous, and using the fact that projection onto $P_{n}$ is continuous, these properties carry to the extension. By $(4), h_{1}$ is a homeomorphism onto $Y$. Notice that $\left\{x \in X \mid w\left(x-x_{1}\right)<w\left(x_{1}\right) / 2\right\}$ is open and shrinkable in $X$ with respect to the original topology, and that the boundary of $C \cap X$ in $X$ is $\left(\mathrm{Bd}_{w} C\right) \cap X$. Since the distance $d_{1}$ is also continuous in the given topology, our argument proves that $h_{1} \mid X$ is a homeomorphism of $X$ onto itself.

If $y \in\left[y^{\prime}, y^{\prime \prime}\right]$ with $y^{\prime} \in P_{n}, y^{\prime \prime}=y^{\prime}+\lambda x_{1}$, then $h_{1}(y)=y$ if $h_{1}\left(y^{\prime}\right)=y^{\prime}$, $h_{1}\left(y^{\prime \prime}\right)=y^{\prime \prime}$; and $h_{1}(y)=y+x_{1}$ if $h_{1}\left(y^{\prime}\right)=y^{\prime}+x_{1}, h_{1}\left(y^{\prime \prime}\right)=y^{\prime \prime}+x_{1}$. Using the se facts it is not hard to show $d(y, A) \leq 10 w\left(x_{2}\right)$ implies $h_{1}(y)=y+x_{1}$, and $d(y, A) \geq 10 w\left(x_{1}\right)$ implies $h_{1}(y)=y \cdot h_{2}$ will be defined in the same way as $h_{1}$, moving points in direction $x_{2}$, fixing those outside a $10 w\left(x_{2}\right)$ neighborhood of $A+x_{1}$, and translating a $10 w\left(x_{3}\right)$ neighborhood $V$ of $A+x_{1}$ to $V+x_{2}$. Home omorphisms $h_{3}, h_{4}, \cdots$ are similarly defined, and since each point in $X \backslash A$ has a neighborhood on which $\left\{h_{n} \cdots h_{2} h_{1}\right\}$ is eventually fixed, we may define a homeomorphism $h: X \backslash A \rightarrow X$ by $h=\cdots h_{3} h_{2} h_{1}$. It is not hard to see that the range of $h$ is all of $X$.

An isotopy theorem such as Klee's in [3] may also be stated in this context. To see this let $h_{0}=\mathrm{Id}$, and define for $x \in X, t \in\left[1-2^{-n}, 1-2^{-n-1}\right]$, $f_{t}(x)=\phi(j(x))$, where

$$
\phi(x)=x+\frac{t-1+2^{-n}}{2^{-n}-2^{-n-1}}\left(h_{n+1}(x)-x\right) \quad \text { and } \quad j(x)=b_{n} \cdots h_{2} h_{1} h_{0}(x) .
$$

$\phi$ is $1-1$, since $h_{n+1}$ is monotone increasing on each line in direction $x_{n+1}$. By (4), $\phi$ is a homeomorphism onto $X$, so that $f_{t}$ is also. Notice that $f_{0}=\mathrm{Id}$. Letting $f_{1}(x)=h(x)$ for each $x \in X \backslash A$ and $H(x, t)=\left(f_{t}(x), t\right)$, we check that $H$ is an invertible is otopy. $H$ maps $X \times\left[1-2^{-n}, 1-2^{-n-1}\right]$ onto itself in a continuous 1-1 fashion. $H(x, t)-(x, t)=\left(f_{t}(x)-x, 0\right)$ lies in the compact subset 


$$
\left(\left[0, z_{2}\right] \cup\left[z_{2}, z_{3}\right] \cup \ldots \cup\left[z_{n+1}, z_{n+2}\right]\right) \times\{0\}
$$

of $X \times R$, so (4) shows $H \mid X \times\left[1-2^{-n}, 1-2^{-n-1}\right]$ is a homeomorphism. We thus have $H \mid X \times[0,1)$ is a homeomorphism of $X \times[0,1)$ onto itself, and it is a routine matter to check the continuity of $H$ at points $(x, 1)$, with $x \in$ $X \backslash A$, and of $H^{-1}$ at points $(x, 1), x \in X$.

\section{REFERE NCES}

1. C. Bessaga, Negligible sets in linear topological spaces, Bull. Acad. Polon. Sci. Ser. Sci. Math. Astronom. Phys. 16 (1968), 117-119. MR 37 \#1946.

2. R. T. Ives, Semi-convexity and locally bounded spaces, Ph.D. Thesis, University of Washington, Seattle, Wash., 1957.

3. V. L. Klee, Convex bodies and periodic homeomorphisms in Hilbert space, Trans. Amer. Math. Soc. 74 (1953), 10-43. MR 14, 989.

4. - Shrinkable neighborhoods in Hausdorff linear spaces, Math. Ann. 141 (1960), 281-285. MR 24 \#A1003.

5. C. A. Riley, Negligibility in nonlocally convex spaces, Proc. Amer. Math. Soc. 41 (1973), 619-624.

DEPARTMENT OF MATHEMATICS, KEENE STATE COLLEGE, KEENE, NEW HAMPSHIRE 03431 